\title{
Deviation from flatness of surfaces after combined peripheral grinding
}

\author{
Vladimir G. Gusev ${ }^{1, *}$, and Alexey V. Morozov ${ }^{1}$ \\ ${ }^{1}$ Institute of Engineering and Automobile Transport, Vladimir State University, Gorky street 87, \\ Vladimir, 600000, Russian Federation
}

\begin{abstract}
A new method of combined flat peripheral grinding is examined, which allows to carry out simultaneously preliminary and final machining of the planes without replacing the coarse with the fine-grain grinding wheel, to eliminate the auxiliary time for the tool replacement, to correct it and set the grinding equipment in order to accomplish the given size. An experimental multifactor model which relates the surfaces flatness deviation with independent factors of the combined grinding process is established on the basis of which it is possible to assign the cutting conditions that provide the required flatness with the greatest possible longitudinal and cross-feed of the blank.
\end{abstract}

The deviation from planeness of surfaces of the details forming assembly unit are making a great influence on operate reliability of cars and mechanisms. High demands to planeness are shown for directing cutting machines, case details of the process equipment, etc. For today accuracy and productivity of grinding processes [1], a roughness of a surface [2], models of a surface on the basis of the stochastic description of the tool properties [3], and also formation grounded surfaces [4] are investigated. Are investigated also grinding processes by highly porous wheels [5], wheels with discrete peripheral [6] and a face [7] cutting surface. Discrete tools are characterised by specific dynamics of formation and heat removal, formation of geometry of the processed surface, self-regulation of a profile of a cutting surface, etc. [8]. The technology of a high-frequency discretization of a cutting surface has been developed for peripheral tools by use of highly concentrated energy flows (a laser beam and a hydro-abrasive stream of a high pressure) [9]. Highly porous and discrete grinding wheels provide considerable decrease in thermal stress level of processes, raise of productivity and physics-mechanical characteristics of the processed blanket [5-7]. Together with it named above research do not mention a question of decrease in an auxiliary time at performance of preliminary and definitive grinding, that reduces productivity of machining processes.

The purpose of the present research consists in working out of a way of the combined peripheral grinding of the planes [10], characterised by higher productivity of a production operation at maintenance of demanded values of a deviation from planeness of processed surfaces. To achieve the objective the following problems are solved:

\footnotetext{
*Corresponding author: prof gusev@mail.ru
} 
- to develop new a way of the peripheral grinding, allowing to spend simultaneously preliminary and definitive machining of planes on one rig without replacement of a coarsegrained wheel by the fine-grained;

- to plan and realise the experiment, allowing to instal the multifactorial models, connecting independent factors of process of combined grinding with parametre of optimisation - a deviation from planeness of surfaces:

- geometrically to interpret the gained multifactorial models, therefore to gain $3 D-X Y Z$ a surface-drawing and $3 D-X Y Z$ the contours-drawing, quantitatively characterising parametre of optimisation as entrance independent factors;

- to develop well-founded recommendations to destination regimes of the combined grinding, providing demanded values of a deviation from planeness of the processed surfaces at the greatest possible productivity of machining.

The tool for the combined grinding consists of fine-grained 1, the coarse-grained 2 grinding wheels, fixed on a spindle 3, which is rotated on arrow $\operatorname{Dr}$ (Fig. 1,a).

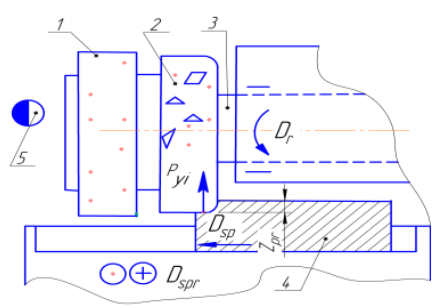

$a$

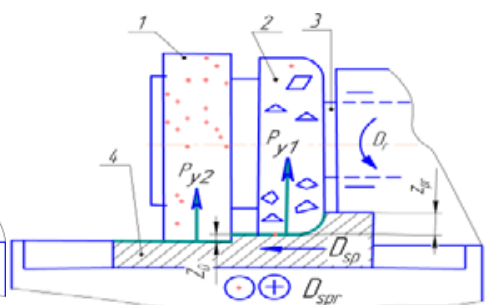

$b$

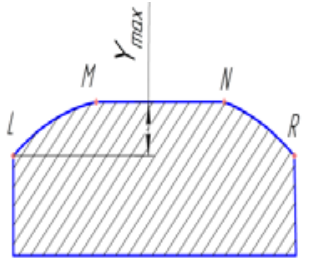

$c$

Fig. 1. Workpiece machining by coarse-grained wheel $(a)$, by the coarse also fine-grained wheels $(b)$ and the form of a surface after grinding process $(c)[10]$.

Before machining the workpiece 4 is located behind the coarse-grained wheel 2 (see from the position of the worker 5 , who is operating the machine tool), which begins the grinding operation by the first. The radial component $P y$ of cutting force acts and bends a spindle. At included longitudinal and cross-feed in the direction of the arrows Dspr and Dsp increases the contact square of workpiece 4 with the coarse-grind wheel 2 , hence, the cutting force and elastic displacements of the spindle also increases during the grinding time. Then, the fine-grain grinding wheel 1 (Fig. 1,b) also enters into cutting process, what causes a further increment of the force Pyi and the mentioned elastic displacements. When both wheels are in the work, the elastic displacements of the spindle reach a maximum value, but at the decrease of contact square of workpiece with the grinding wheels 1 and 2 , they gradually become equal to zero.

As a result of the change of the elastic displacements, the machined surface represents a convex $L M N R$ contour with a flatness deviation equal to:

$$
Y \max =\{P y 1+P y 2\} / J_{s},
$$

where $P y 1, P y 2$ - the radial component of cutting force, created by the work of coarse and fine-grain wheel, respectively; $J_{S}$ - is the stiffness of the spindle with the installed grinding wheels in perpendicular direction to the machined surface.

The tool for the combined grinding allows to carry out additional technological pass, in which finish machining is carried out by fine-grained wheel at move of workpiece in direction from the operator 5. That the coarse-grained wheel did not damage the definitively processed surface, diameter $\mathrm{Dpr}$ of its cutting surface should be less diameter Do of a fine-grained wheel. Minimum values of flatness deviation and of other quality 
indicators of the surface layer are providedm when the fine-grained wheel performs the final pass with an allowance, equal to:

$$
\text { to }=Y \max +R \max +T,
$$

where Ymax, Rmax, $T$ - accordingly a deviation from planeness, the maximum microgeometry and depth of a defective layer, which have been generated at grinding by coarse-grained wheel.

The maximum microgeometry Rmax and the depth $T$ of the defective layer are determined by using depending on the applied characteristic of abrasive material of the coarse-grinded wheel, regime of its editing and the cutting regime. The deviation from planeness Ymax can be calculated at a known grinding regime, force loading on an edge tool, workpiece, and a spindle rigidity. Combined grinding is characterized by a higher productivity by comparison with the traditional grinding, because it allows to exclude or substantially reduce the auxiliary time for technological operation: the replacement of the coarse by the fine-grain grinding wheel, its truing and the adjustment of the machining tool for a size and etc. For definition of technological possibilities of the combined grinding a multifactorial experiment on the surface-grinding machine $3 G 71 \mathrm{M}$ has been realised.

The preliminary grinding was performed with a coarse grinding wheel PP $250 * 76 * 20$ 25AF46L8V5A2 GOST R52381-2005, GOST R52587-2006, and the final grinding with a $P P 250 * 76 * 1025 \mathrm{~A} F 90 K 8 V 5 A 2$ on a cutting speed of $35 \mathrm{~m} / \mathrm{s}$. During the traditional machining, the same machine tool, the same cutting regimes and grinding wheels were used, but after the preliminary machining with the coarse grinding wheel, this one was replaced by the fine-grain grinding wheel. Workpieces-plates with dimensions $100 \cdot 100 \cdot 30$ $\mathrm{mm}$ from steel $30 H G C A$ with hardness $H B=29,0-35,5$ by using of a coolant of a $5 \%$ aqueous solution of LACTVCA WBA 5400 were machined. The flatness deviation of the ground surface has been measured by device IGP GOST 28798-90. The plan of the full factorial experiment $\mathrm{N}=2^{3}=8$ was realized. Independent factors were chosen: allowance thickness $t$, taken off for the double course of a workpiece; longitudinal Spr and cross $S p$ feed of the workpiece. For optimization parameter the deviation from planeness of surfaces, subjected to the combined grinding is accepted. As a result of the statistical data processing, some regression equations were obtained, which reflect the influence of each one of the factors on the optimization parameter:

$$
\begin{gathered}
\Delta p l=2,80+330 * t, \\
\Delta p l=2,63+0,39 * S p r, \\
\Delta p l=5,5+0,167 * S p,
\end{gathered}
$$

where $\Delta p l-$ is the natural designation of the optimization parameter; $t, S p r, S p-$ are the natural designation of the factors.

The interactive influence of the factors $t$ and $S p r$ on the parameter $\Delta p l$ is described by the regression equation:

$$
\Delta p l=0,105+305 * t+0,37 * S p r .
$$

The graphical interpretation of the model (6) is presented by the $3 D-X Y Z$ surface-graph (Fig. 2), in which the values of the factors $t$ and $S p r$ are represented in the horizontal plane, and the flatness deviation values of the ground surfaces are indicated vertically. To the right of a response surface (a colour inclined plane) six rectangles with the numbers reflecting 
values of a deviation from planeness are presented. The minimum values of the parameter $\Delta p i<2 \mathrm{mkm}$ are typical for the dark green rectangle (the bottom rectangle in a column), and the values $\Delta p i>10 \mathrm{mkm}$ for the top rectangle of dark brown colour. The response surface has colours similar to the colours of the rectangles, what allows to determine the deviation from flatness $\Delta p l$ of the surfaces for specific values of the removed allowance $t$ and the longitudinal feed $S p r$ of the workpiece. These experimental data are necessary for well-founded appointment of a regime of the combined grinding depending on demands to the planeness limited by the drawing of a product.

The interactive influence of the thickness $t$ of the allowance and the cross-feed $S p$ on the flatness deviation is described by the regression equation:

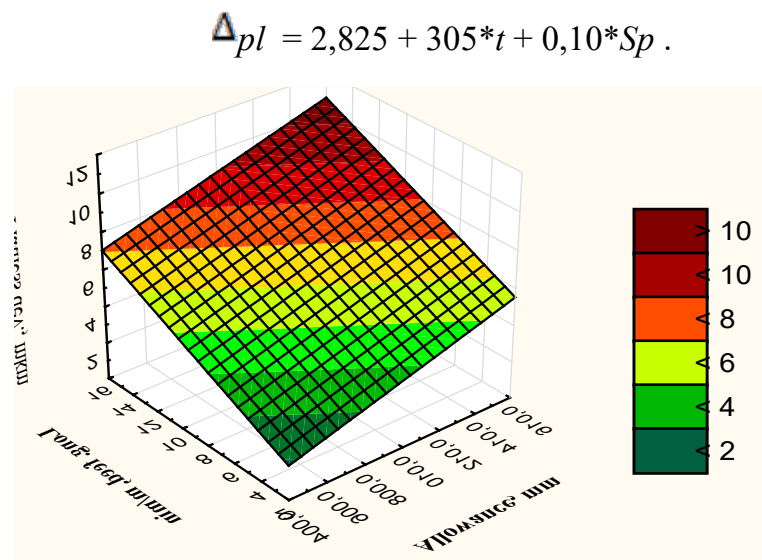

Fig. 2. 3D-XYZ surface-graph of the interactive influence of the allowance $t$ and longitudinal feed $S p r$ of the workpiece on the optimization parameter.

The graphical interpretation of the multifactor model (7) is presented by the $3 D-X Y Z$ surface-graph (Fig.3). The column to the right of the response surface contains five rectangles. They evidence about a smaller dispersion of the flatness deviation of the ground surface in a comparison with the multi-factor model (6). The interactive influence of the longitudinal $S p r$ and cross-feed $S p$ on the parameter $\Delta p l$ of the ground surface is described by the regression equation:

$$
\Delta p l=2,545+0,37 * S p r+0,1 * S p .
$$

The graphical interpretation of model (8) is presented by the $3 D-X Y Z$ surface-graph (Fig. 3). The comparison of (6) - (8) and 3D-XYZ graphics-surfaces (Fig. 1) - (Fig. 3) shows, that the longitudinal feed has a greatest influence on the flatness deviation of surfaces, subjected to the combined grinding. The cross-feed $S p$ of workpiece influences on the parameter $\Delta p l$ in a greater degree, than a removed allowance $t$, but in smaller degree, than a longitudinal feed $S p r$. A growth of independent factors $S p r, S p$ and $t$ leads to a deeper introduction of cutting abrasive grains of a coarse-grained wheel into a metall. Under the influence of the force load the sharp peaks of the abrasive grains are rounded off. 


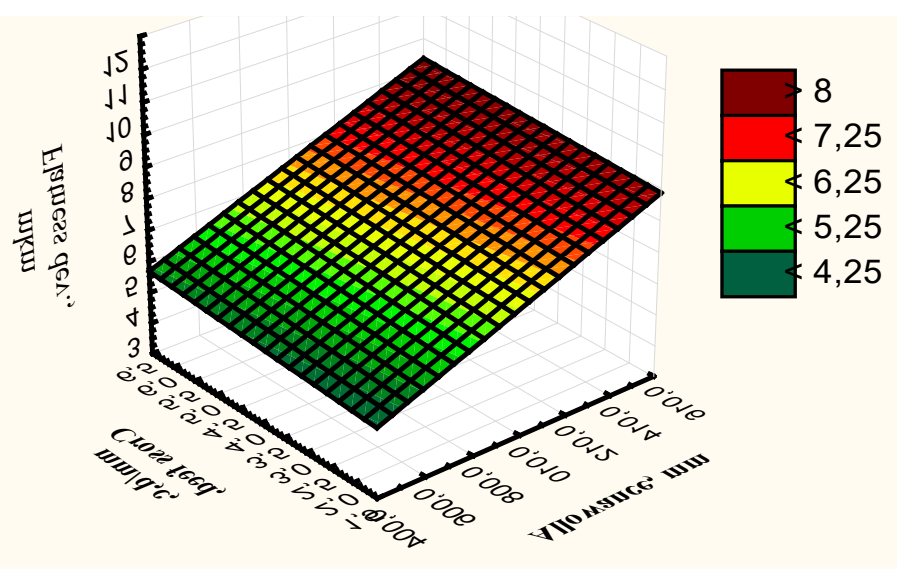

Fig. 3. 3D- $X Y Z$ surface-graph of the interactive influence of the thickness of the removed allowance $t$ and the cross-feed $S p$ on the flatness deviation. $\Delta p l$

The wear and waviness of the cutting surface of the wheel during grinding are increased. The changed initial geometry of the cutting surface of the coarse grinding wheel is copied onto the machined surface, causing an increasing of the flatness deviation. $\Delta p l$ $3 D-X Y Z$ projections-graphs (Fig. 5, a-c) are very informative and important for process engineers, who design new finishing processes. On the basis of $3 D-X Y Z$ projections-graphs a valid choice of the combined grinding mode is carried out. The process engineer, projecting operation of combined grinding, on the value of parameter $\Delta p l$ indicated in the drawing of a detail, chooses a rectangle, which colour corresponds to the set deviation from planeness, then on value of a thickness of the allowance, which is subject to removal, defines on an axis of ordinates (fig. 5, and,) the value of longitudinal and cross-section feed of workpiece. Further he finds crossing of values of longitudinal and cross-section feed (Fig. 5,), thus a colour of the strip, corresponding to crossing of named feeds, should coincide with colour of an initial rectangle.

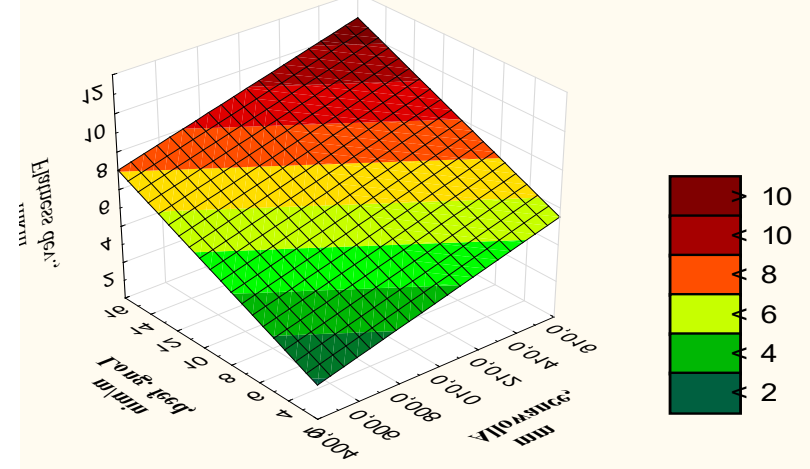

Fig. 4. 3D-XYZ surface-graph of the interactive influence of the longitudinal $S p r$ and cross-feed $S p$. 


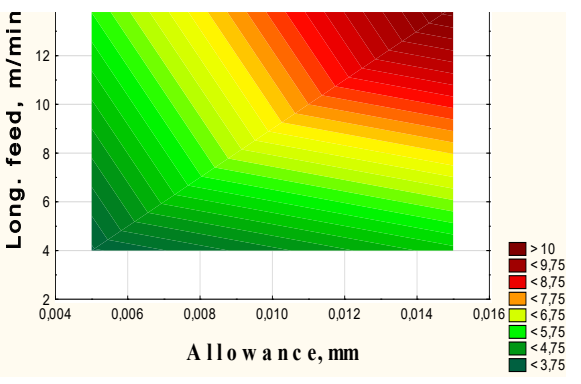

$a$

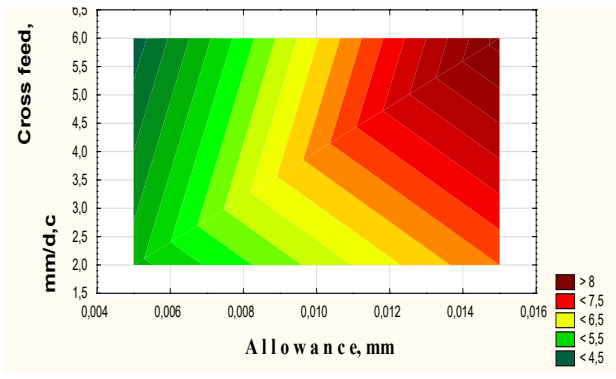

$b$

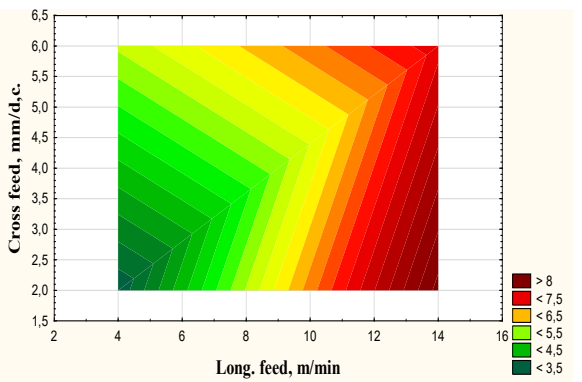

C

Fig. 5. 3D-XYZ projection-graphs of the interactive influence of allowance $t$ and longitudinal feed $\operatorname{Spr}(a)$; cross-feed $S p$ and allowance $t(b)$; longitudinal $S p r$ and cross-feed $S p(c)$ on the flatness deviation $\Delta p l$ of the surfaces.

The last process is performed to verify the correctness of the selected workpiece feeds, and for that, the regression equations (6) - (8) can also be used. As appears from the above, appointment of a mode of the combined grinding occupies insignificant time, thus a mode provides a deviation from planeness of the processed surface, set by the drawing. The comparison of the optimization parameter of the surfaces, machined by the traditional and proposed processe, showed, that after the manufacturing step by a combined tool the flatness deviation more on (6-8) \%. It is explained by the perturbations of the fine-grain grinding wheel from the side of coarse-grain wheel. If drawing demands to optimization parameter $\Delta p l$ are not observed, so a final technological step should be executed at use only of a fine-grained grinding wheel. In this case, the deviation from planeness of the surfaces, processed by traditional and proposed technology, is analogous.

Thus, combined grinding does not concede traditional one according to the flatness deviation parameter and simultaneously allows to exclude or considerably to reduce the auxiliary time for performance of technological operation, what leads to increase of machining productivity.

\section{References}

1 I.I. Koltunov, Iu.S. Stepanov, A.S. Tarapanov. Raise of accuracy and quality of grinding of internal curvilinear surfaces. Moscow, 270 (2007).

2 Yu. K. Novoselov, V. Bogutsky, L.Shron, and A. Kharchenko. Forecasting the surface roughness of the workpiece in the round external grinding. MATEC Web of Conferences 129 (2017).

3 Yu.K. Novoselov, D.A. Kainov, S.M. Bratan, The construction of the model of a ground surface on the basis of a stochastic description of the properties of the tool, 
Nauk. pr. Donec. nac. tehn. un-tu. Ser.: Mashinoobladnannja, Issue 7. PP.17-31 (2010).

4 Iu.K. Novoselov, Dynamics of surfaces forming at abrasive machining, Sevastopol', (2012).

5 V.K. Starkov, Grinding by high-porous wheels. Moscow, 688 (2007).

6 V.G. Gusev, A.V. Morozov. Flat Peripheral Grinding with Discrete Wheels. The R.F, Yoshkar-Ola, 222 (2012).

7 A.V. Morozov, V.G. Gusev. Discrete Plane Face Grinding, Moscow, Pen Publishing House, 124 (2016).

8 V.G. Gusev, A.V. Morozov, P.S. Shvagirev, Evaluating discrete wheels and their influence on grinding dynamics, J. Sci. Russian Engineering Research. 29(8) (2009) 835-837.

9 V.G. Gusev, A.V. Morozov, Discretization technology of abrasive wheels operating surfaces with laser and hydro-abrasive jet, J. Sci. Science Intensive Technologies in Mechanical Engineering. 9(73) (2017) 20-27.

10 V.G. Gusev, A.V. Morozov. The R.F. Patent 2,606,143. (2017) 